\title{
Influence of Array Configuration on MIMO Channel Capacity
}

\author{
C. Gómez-Calero, R. Martínez-Rodríguez-Osorio \\ Radiation Group, Universidad Politécnica de Madrid \\ E-mail: carlosgc@gr.ssr.upm.es
}

\section{Introduction}

The use of multiple antennas at each side of the radio link has demonstrated an increase in terms of data bit rate and spectral efficiency [1]. Due to this, different techniques such as spatial multiplexing and space-frequency-time techniques are being applied to MIMO (Multiple-Input Multiple-Output) systems. In order to obtain MIMO performances, most of the research studies have evaluated Uniform Linear Arrays (ULAs) for simulations and measurements. However, other considerations can be taken into account with the similar space used for the transmitter or receiver antenna array using different physical configurations. In this work, several antenna array configurations in the transmitter and the receiver have been studied and compared, in terms of MIMO channel capacity, showing that for a limited space, the linear array could be replaced for a rectangular or circular one with similar performances.

\section{MIMO Channel Model}

The MIMO channel model used for the study has been the 3GPP-3GPP2 Spatial Channel Model (SCM) [2]. This 2-D propagation-based model includes physical parameters in the simulation but also with a stochastic basis. Due to the channel model is suitable for outdoor scenarios, it is necessary to change the main parameters of the simulation for indoor scenarios. The cell radius is $100 \mathrm{~m}, 3$ and $1.5 \mathrm{~m}$ of height for the transmitter and receiver, $180^{\circ}$ of maximum Angle of Departure (AoDmax) and Mobile Station speed is $1 \mathrm{~m} / \mathrm{s}$. Once the wideband channel matrix $H(t, \tau)$ is obtained from the $\mathrm{SCM}$, an equivalent narrowband channel matrix is computed for $f_{o}=2.45 \mathrm{GHz}$, given by (1).

$$
H(t)=\sum_{n=1}^{L} H\left(t, \tau_{n}\right) e^{\left(-j \cdot 2 \pi \cdot f \cdot \tau_{n}\right)}
$$

The MIMO channel capacity is calculated by (2), where $\mathbf{I}_{\mathrm{MR}}$ is the eye matrix, $\rho$ represents the signal to noise ratio, $\mathrm{M}_{\mathrm{T}}$ indicates the number of transmitter antennas, $\mathbf{M}_{\mathrm{R}}$ the number of receiver antennas, $\mathbf{H}$ is the channel matrix including antenna radiation patterns, and there is no Channel State Information (CSI) in the transmitter.

$$
C=\log _{2}\left|\mathbf{I}_{M_{R}}+\frac{\rho}{M_{T}} \mathbf{H H}^{H}\right| \mathrm{bps} / \mathrm{Hz}
$$




\section{MIMO Array Configurations}

Once the channel parameters are defined, they are used to generate the channel coefficients. For an $s$ element linear BS array and a $u$ element linear MS array, the channel coefficients for one of $n$ multipath components are given by (3)

$$
h_{u, s, n}(t)=\sqrt{\frac{P_{n} \sigma_{S F}}{M}} \times \sum_{m=1}^{M}\left(\begin{array}{l}
\left.\left[\begin{array}{c}
\chi_{B S}^{(v)}\left(\theta_{n, m, A o D}\right) \\
\chi_{B S}^{(h)}\left(\theta_{n, m, A o D}\right)
\end{array}\right]^{T}\left[\begin{array}{cc}
\exp \left(j \Phi_{n, m}^{(v, v)}\right) & \sqrt{r_{n 1}} \exp \left(j \Phi_{n, m}^{(v, h)}\right) \\
\sqrt{r_{n 2}} \exp \left(j \Phi_{n, m}^{(h, v)}\right) & \exp \left(j \Phi_{n, m}^{(h, h)}\right)
\end{array}\right]\left[\begin{array}{c}
\chi_{M S}^{(v)}\left(\theta_{n, m, A O A}\right) \\
\chi_{M S}^{(h)}\left(\theta_{n, m, A o A}\right)
\end{array}\right] \times\right) \\
\zeta_{B S}\left(\theta_{n, m, A o D}\right) \times \zeta_{M S}\left(\theta_{n, m, A O A}\right) \times \exp \left(j k\|\mathbf{v}\| \cos \left(\theta_{n, m, A o A}-\theta_{v}\right) t\right)
\end{array}\right)
$$

where polarization response of each antenna, $\chi(\theta)$, has been considered, and cross polarization discrimination channel is represented by $1 / r_{n}$. However, only the results for a linear array are obtained. In order to include different array configurations, the $\zeta(\theta)$ factor in (3), which represents the phase relative to each antenna element, has been modified to select the type of configuration needed at the transmitter or receiver side: linear (4), rectangular (5) or circular (6).

$$
\begin{aligned}
& \zeta\left(\theta_{n, m}\right)=\exp \left(j k d \sin \left(\theta_{n, m}\right)\right) \\
& \left.\zeta\left(\theta_{n, m}\right)=\exp \left(j \mid k d_{x} \sin \left(\theta_{n, m}\right)+k d_{y} \cos \left(\theta_{n, m}\right)\right]\right) \\
& \zeta\left(\theta_{n, m}\right)=\exp \left(j\left[k R \sin \left(\theta_{n, m}-i \alpha\right)\right]\right)
\end{aligned}
$$

Several configurations have been evaluated for the cases of a 4x4 and 9x9 MIMO channels. For all these situations, both the transmitter and receiver antennas are half a wavelength dipoles. In Figs. 1 to 4 , the linear and rectangular configurations for the $4 \times 4$ MIMO channel are shown. In this case, a circular array offers the same results as configuration 2 and 4, so it has been neglected. On the other hand, for the 9x9 MIMO channel case, configuration 1, 5 (Fig. 5) and 6 (Fig. 6) have been studied. The aim of using 9 antennas is to evaluate a matrix of antennas in a rectangular array. On the other hand, for each configuration, $d x$ and $d y$ are $\lambda / 2$ and $\lambda$, except for the configurations 5 and 6 , where $R=d x=d y=\lambda / 2, \lambda, 2 \lambda$ and $3 \lambda$, and $\alpha=2 \pi / M$. Thus, there are 8 configurations for $4 \times 4$ and 10 for $9 \times 9$ MIMO scheme.

\section{Results}

Table 1 and Table 2 represent the average capacity of all the possible configurations for the transmitter and receiver antenna array for the case of $4 \times 4$ and 9x9 channel, respectively, using SCM implementation in Matlab [3]. In the first case, the best performances are obtained with more spacing between elements in the linear configuration (Tx 1b). It is worth to mention that in case the spacing for the antenna array is limited in $x$ axis (for example, a wavelength), placing the antennas in a rectangular distribution (Tx $2 b, 3 b$ or $4 b)$ offers higher capacity. For WLAN applications $\lambda$ is $12.25 \mathrm{~cm}$, and access points can take the space in advantage to select the best array configuration (Tx 6d). 


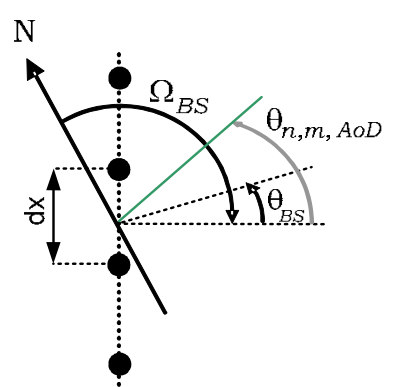

Fig. 1.- Configuration 1

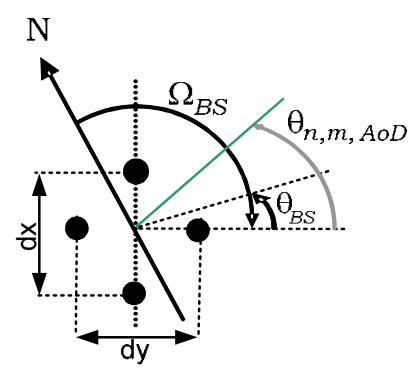

Fig. 4.- Configuration 4

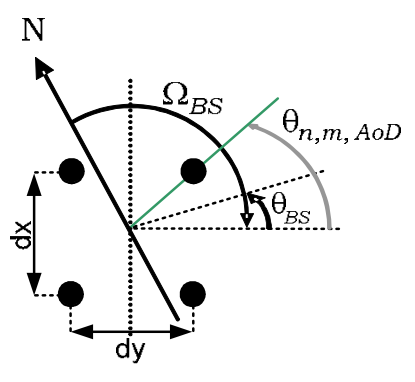

Fig. 2.- Configuration 2

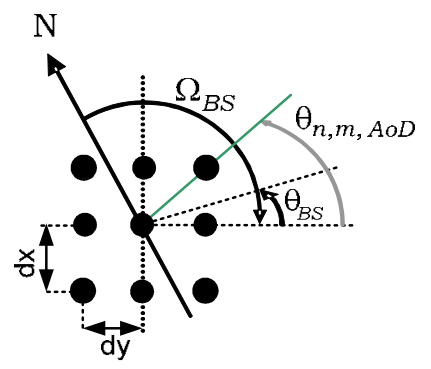

Fig. 5.- Configuration 5

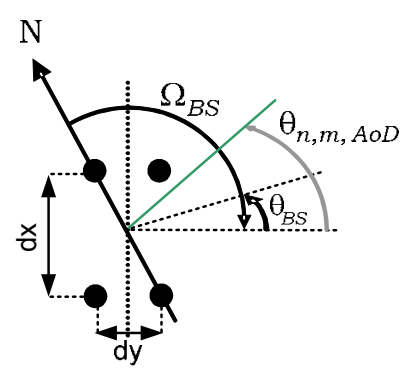

Fig. 3.- Configuration 3

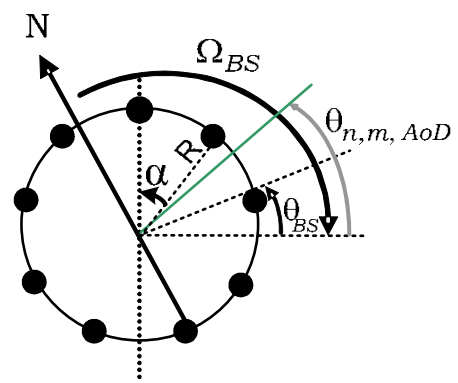

Fig. 6.- Configuration 6

\begin{tabular}{|c|c|c|c|c|c|c|c|c|}
\hline & Rx 1a & Rx 1b & $\mathbf{R x} 2 \mathbf{a}$ & $\mathbf{R x} 2 b$ & $\mathbf{R x} 3 \mathbf{a}$ & $\mathbf{R x} 3 \mathbf{b}$ & $\mathbf{R x} 4 \mathbf{a}$ & $\mathbf{R x} 4 \mathbf{b}$ \\
\hline Tx 1a & 10,10 & 10,30 & 9,90 & 10,30 & 10,10 & 10,10 & 9,90 & 10,10 \\
\hline Tx 1b & 11,72 & 11,92 & 11,11 & 11,72 & 11,31 & 11,31 & 11,11 & 11,52 \\
\hline Tx 2a & 9,49 & 9,70 & 9,29 & 9,49 & 9,29 & 9,29 & 9,29 & 9,29 \\
\hline Tx 2b & 10,91 & 11,11 & 10,51 & 10,91 & 10,71 & 10,71 & 10,51 & 10,71 \\
\hline Tx 3a & 10,10 & 10,30 & 9,90 & 10,10 & 9,90 & 9,90 & 9,90 & 10,10 \\
\hline Tx 3b & 10,30 & 10,30 & 9,90 & 10,30 & 10,10 & 10,10 & 9,90 & 10,10 \\
\hline Tx 4a & 8,69 & 8,89 & 8,48 & 8,69 & 8,69 & 8,69 & 8,48 & 8,69 \\
\hline Tx 4b & 10,10 & 10,30 & 9,90 & 10,30 & 10,10 & 10,10 & 9,90 & 10,10 \\
\hline
\end{tabular}

Table 1.- Capacity results (in bps/Hz) for the case of $4 \times 4$ MIMO scheme

\begin{tabular}{|c|r|r|r|r|r|r|r|r|r|r|}
\hline & Rx 1a & Rx 1b & Rx 5a & Rx 5b & Rx 5c & Rx 5d & Rx 6a & Rx 6b & Rx 6c & Rx 6d \\
\hline Tx 1a & 19,90 & 20,10 & 18,48 & 19,90 & 20,30 & 20,30 & 18,89 & 19,70 & 20,30 & 20,30 \\
\hline Tx 1b & 24,34 & 24,95 & 21,72 & 24,14 & 24,95 & 24,95 & 22,53 & 23,94 & 24,95 & 25,15 \\
\hline Tx 5a & 16,06 & 16,26 & 15,45 & 16,06 & 16,26 & 16,26 & 15,45 & 15,86 & 16,26 & 16,26 \\
\hline Tx 5b & 20,10 & 20,51 & 18,69 & 20,10 & 20,30 & 20,51 & 19,09 & 19,70 & 20,51 & 20,51 \\
\hline Tx 5c & 22,73 & 23,33 & 20,71 & 22,73 & 23,13 & 23,33 & 21,31 & 22,32 & 23,33 & 23,33 \\
\hline Tx 5d & 24,34 & 24,95 & 21,72 & 24,14 & 24,75 & 24,95 & 22,73 & 23,74 & 24,75 & 24,95 \\
\hline Tx 6a & 14,85 & 15,05 & 14,44 & 14,85 & 15,05 & 15,05 & 14,44 & 14,85 & 15,05 & 15,05 \\
\hline Tx 6b & 19,09 & 19,29 & 17,88 & 19,09 & 19,29 & 19,29 & 18,28 & 18,89 & 19,29 & 19,49 \\
\hline Tx 6c & 23,33 & 23,94 & 21,31 & 23,33 & 23,74 & 23,94 & 21,92 & 22,93 & 23,94 & 23,94 \\
\hline Tx 6d & 25,56 & 26,16 & 22,73 & 25,35 & 25,96 & 26,16 & 23,74 & 24,95 & 26,16 & 26,16 \\
\hline
\end{tabular}

Table 2.- Capacity results (in bps/Hz) for the case of $9 \times 9$ MIMO scheme 
In the second case, the 9x9 MIMO scheme, for a limited space in $x$ axis, higher capacity is obtained with a non linear array configuration. For the case of $2 \lambda \times 2 \lambda$ of space, the best performances are given by the rectangular configuration $5 \mathrm{~d}$. This configuration offers similar results than in the case of a linear array with a length of $3 \lambda$. On the other hand, Fig. 7 and Fig. 8 represent the cumulative distribution function of the capacity for the case of using the configuration $1 \mathrm{~b}$ at the transmitter, where similar curves are obtained in the $4 \times 4$ case for all the configurations, however for the case of 9x9, the same transmitter yields high capacity dispersion for all the receivers.

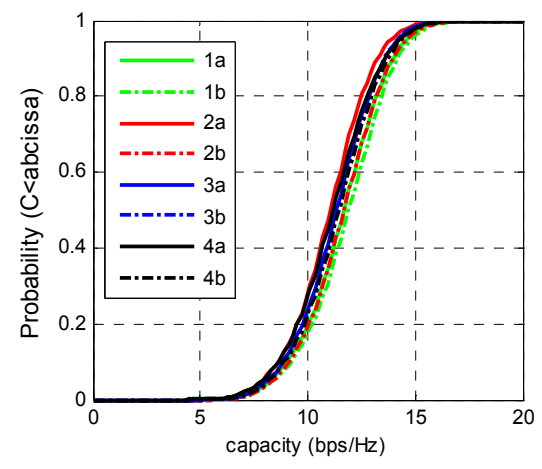

Fig. 7.- CDF of capacity for $4 \times 4$ case

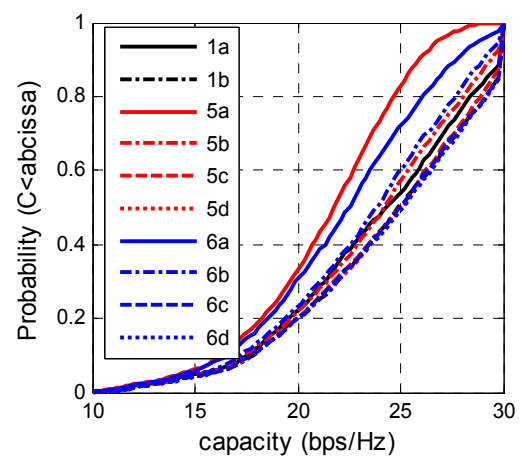

Fig. 8.- CDF of capacity for 9x9 case

\section{Conclusions and future work}

A study of the influence of antenna array configuration in the MIMO channel performances has been carried out. Two cases (4x4 and 9x9) of MIMO schemes have been taken into account including polarization in the channel model (SCM). For each case, several configurations have been evaluated, including linear, bidimensional and circular arrays. Results show that for limited space in one axis in the transmitter or the receiver, rectangular and circular array offer better performances. This is quite interesting for base stations even user terminals antenna arrays. In this study dipoles have been used, although other types of antennas based on PIFAs [4] are currently being evaluated and measured.

\section{References:}

[1] G.J. Foschini and M.J. Gans, "'On Limits of Wireless Communications in a Fading Environment when Using Multiple Antennas", Wireless Personal Communications, no. 6, pp. 311-335, 1998 .

[2] 3rd Generation Partnership Project, "Spatial channel model for Multiple Input Multiple Output (MIMO) simulations", Technical Specification Group Radio Access Network, 3GPP TR 25.996 V6.1.0 (2003-09).

[3] J. Salo, G. Del Galdo, J. Salmi, P. Kyösti, M. Milojevic, D. Laselva, and C. Schneider. (2005, Jan.) "MATLAB implementation of the 3GPP Spatial Channel Model (3GPP TR 25.996)" [Online]. Available: http://www.tkk.fi/Units/Radio/scm/

[4] C. Gomez-Calero, L. Gonzalez-Diaz, R. Martinez-Rodriguez-Osorio, "Multi-band Planar Inverted-F Antennas for MIMO mobile terminals", 2007 IEEE Antennas and Propagation International Symposium, pp. 2413-2416, June 2007. 